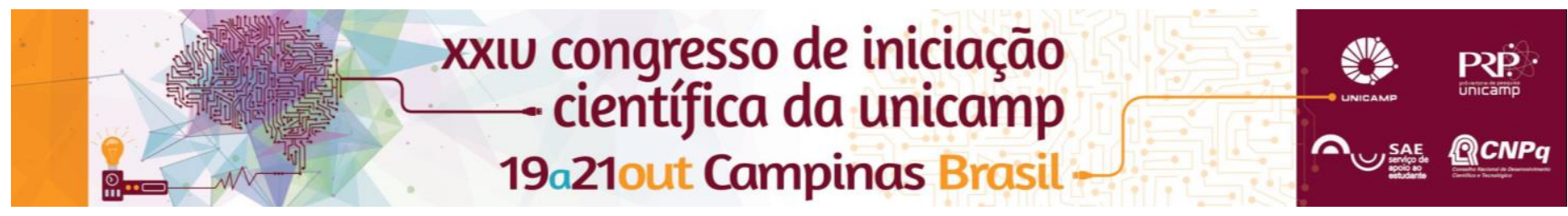

\title{
Influence of cold plastic deformation on phase transformations and mechanical properties in metastable beta-tit anium alloys.
}

\author{
Rodrigo J. Contieri (PQ), Luiz G. Soares (IC).
}

\begin{abstract}
Annealing heat treatment in hardened materials induces the occurrence of phenomena such as recovery, recrystallization and grain growth, resulting in microstructural changes and different properties of the material in levels previous to the deformation. This work evaluated the influence of cold work in the phase transformations and hence the mechanical properties in Ti-15Mo-3Al-0.2Si-3NB, a metastable beta-titanium alloys, and variations with $\mathrm{Cr}$ additions, Ti13Mo-3NB-3Al-0.2Si-1,6Cr and Ti-13Mo-3NB-3Al-0.2Si-3,2Cr when subjected to different deformation and heat treatments. These processes have been investigated by microstructural analysis, differential scanning calorimetry and Vickers microhardness measurements, which allowed the quantitative determination of the activation energy involved in the recrystallization of the material, the recrystallization temperatures, the evolution of the recrystallized fraction with temperature, dislocation density and the driving force associated with the recrystallization and grain growth kinetics.
\end{abstract}

\section{Key words: Metastable Beta-titanium Alloys, Plastic Deformation, Phase Transformation.}

\section{Introduction}

Titanium alloys are technologically relevant materials due to their many possible applications. The claim for materials with high strength-to-weight ratio resulted in larger use of metastable beta-titanium alloys type. The beta phase (BCC) in titanium alloy has low strength and low modulus of elasticity. As an example of application, one can mention their use in chemical and aerospace industries for the manufacturing products ${ }^{1}$.

It is well known that the mechanical properties of titanium alloys are associated with their microstructure, which in turn is a function of composition and mainly, processing conditions. Experiments with TIMETAL 21S commercial alloy show that new processing routes permit to obtain better strength values than those observed in conventional heat treatments applied in industry. Application of annealing heat treatments to strain hardened metallic materials may give rise to recuperation, recrystallization and grain growth phenomena. This heat treatment leads to microstructure transformation and hence, changes in physical and mechanical properties.

An important issue for the development of high strength titanium alloy is about controlling the microstructures in function of process parameters. Then, this work aims at studying the processes of recrystallization and grain growth in cold deformed Ti-15Mo-3Al-0.2Si-3NB, a metastable beta-titanium alloys, and variations with $\mathrm{Cr}$ additions, $\mathrm{Ti}$ 13Mo-3NB-3Al-0.2Si-1.6Cr and Ti-13Mo-3NB-3Al-0.2Si$3.2 \mathrm{Cr}$ using a microstructural analysis, DSC and Vickers microhardness measurements to evaluate the process parameters involved and the kinetics of recrystallization and grain growth.

\section{Results and Discussion}

The addition of chromium in the TIMETAL 21S alloy resulted in a reduction of the grain size and changes in the slip mechanism of the material when subjected to plastic deformation. Consequently, as a metastable material, there was the formation of different phases in accordance with the increase of the deformation rate. As one can see in Image 1, the addition of $\mathrm{Cr}$ made the number of grains much higher. Beyond that, the slip mechanism in the alloy with higher chromium content and in the alloy with $0 \% \mathrm{Cr}$, were the phase transformation, from $\beta$ to martensite and DOI: 10.19146/pibic-2016-51857 the twin formation. While the alloy with $1.6 \% \mathrm{Cr}$ only the formation of the martensite phase happened as expected. These phenomena can be proved with the results of the hardness tests that shows better mechanical properties in the $A$ and $C$ alloys after deformation than in the B alloy.

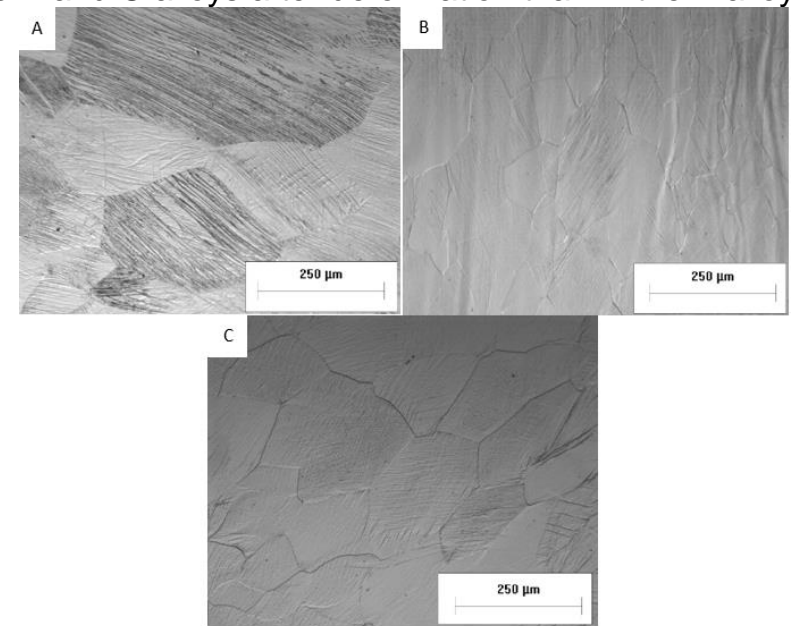

Image 1. Microstructure with $30 \%$ plastic deformation (A$0 \% \mathrm{Cr}, \mathrm{B}-1.6 \% \mathrm{Cr}$ and $\mathrm{C}-3.2 \% \mathrm{Cr}$ )

\section{Conclusions}

The alloy Ti-15Mo-3Al-0.2Si-3NB, a metastable betatitanium alloy and variations with $\mathrm{Cr}$ additions, Ti-13Mo3NB-3Al-0.2Si-1.6Cr and Ti-13Mo-3NB-3Al-0.2Si-3.2Cr, rolled at room temperature at a deformation of $30 \%, 60 \%$ and $90 \%$, showed a strong influence of the deformation and the composition on the slip mechanism, which changed the mechanical properties with the formation of new phases and twins.

\section{Acknowledgement}

School of Applied Sciences (FCA), University of Campinas, Limeira, SP, Brazil and CNPq.

CONTIERI, R. J., ZANOTELlO, M., CARAM, R Recrystallization and Grain Growth in Highly Cold Worked CP-Titanium. Materials Science and Engineering A, Structural Materials: properties, microstructure and processing, p. 1, 2010. 